\title{
Study on the Characteristics of Low-speed Boundary Layer Flow Separation
}

\author{
He Peng ${ }^{\mathrm{a}}$, Li Lei ${ }^{\mathrm{b}}$, Chen Zhongying ${ }^{\mathrm{c}}$, Li Man ${ }^{\mathrm{d}}$ \\ Aviation University of Air Force, Changchun, Jilin, China \\ aHepeng@163.com, 'bi-lei@126.com, 'zhongying@126.com, 'liman2667@sohu.com,
}

Keywords: Low-Speed; Boundary Layer; Viscous Fluid

\begin{abstract}
The boundary layer theory is proposed by German scientist Prandtl. When the fluid motion in the large Reynolds number, a thin layer will be concentration to produce in the fluid surface. Because the thin area is very close to the object surface in which the viscous force plays an important role, which is called the boundary layer. Due to the fact that the fluid is viscous, he will produce some phenomena which are different from the ideal fluid. In this paper, the air viscosity and the flowing phenomena and laws in the low-speed boundary layer are analyzed of the air flow very close to the aircraft surface.
\end{abstract}

\section{Introduction}

In the early days, all of the fluid was considered to be an ideal fluid. Ideal fluid is a kind of ideal model with no viscosity [1] [2]. That is, all of the fluid is not viscous. In nature, all actual fluid is viscous. Water and air are viscous, which can be observed through the river water. The water in the middle of the river flows rapid. The nearer the river bank, the slower the water flows and the water very close to the bank does not basically flow. The reason for this phenomenon is that the bank is not smooth, and the river is viscous. As a result, the river is affected by the friction and its own viscous. So, close to the shore, the water is almost not flowing. From the shore to the center, the influence gradually decreases, the water was gradually speeded up.

Air is also viscous. The experiment models include two discs close to but not touching each other. Above the disc is static, the moment the disc rotation for some time later, the disc is also slowly rotating up. In order to explain the phenomenon, the air is divided into a number of layers, and two adjacent air layers are taken out of the two adjacent layers. The lower the air velocity, the molecular clusters with great momentum, when the irregular thermal motion of air molecules into the upper, through intermolecular mixing and collision momentum increases the upper air parcels, the upper air is accelerated; so in this experiment, the disc when rotating, an air layer close to the surface of the disk with the disk with high-speed rotation.

Such a characteristic of interaction of the two adjacent air layers when the relative motion occurs in the air is called the air viscosity [3].

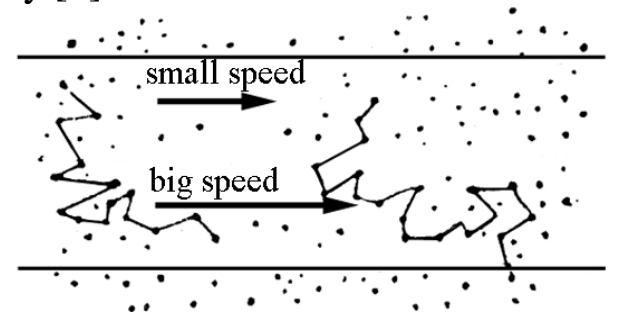

Fig. 1 Two Adjacent Air Layers with the Different Flow velocities

\section{Steady Flow and Unsteady Flow}

The space that the fluid of motion occupies is called the flow field. The study of the fluid motion does not specifically depend on how a certain fluid particle moves but on the analysis of the fluid motion of each fixed space point in the flow field and then on the combination of the changes of parameters of motion of all the space points in the flow field, thus finding the fluid law of motion 
[4].

The position of each fixed point in the flow field can be indicated in the determined rectangular space coordinates $(x, y, z)$ and the law of motion can be described by the fluid state parameters (pressure, density, velocity, etc.) of each point at the different instants ( $t$ ) [5]. Obviously, all the fluid state parameters are the functions of space positions and time as follows:

$$
\begin{gathered}
p=f_{1}(x, y, z, t) \\
\rho=f_{2}(x, y, z, t) \\
V_{x}=f_{3}(x, y, z, t) \\
V_{y}=f_{4}(x, y, z, t) \\
V_{z}=f_{5}(x, y, z, t)
\end{gathered}
$$

$x, y, z$ and $t$ are four independent variables in mathematics, but space variables and time variables are different in physics [6]. The fluid flow in the flow field is in the two forms of steady flow and unsteady flow corresponding to the time variables. It is the steady flow if any fluid state parameter does not change with the time $(t)$ as far as any fixed point in the flow field is concerned; vice versa, it is the unsteady flow if the fluid state parameters change with the time [7]. For example, the water of each point in the middle of the river, whose state parameters do not change with the time, is in the steady flow; the vortex formed behind the bridge pier and the eddy flow at the back of the traveling vehicle are the unsteady flow [8].
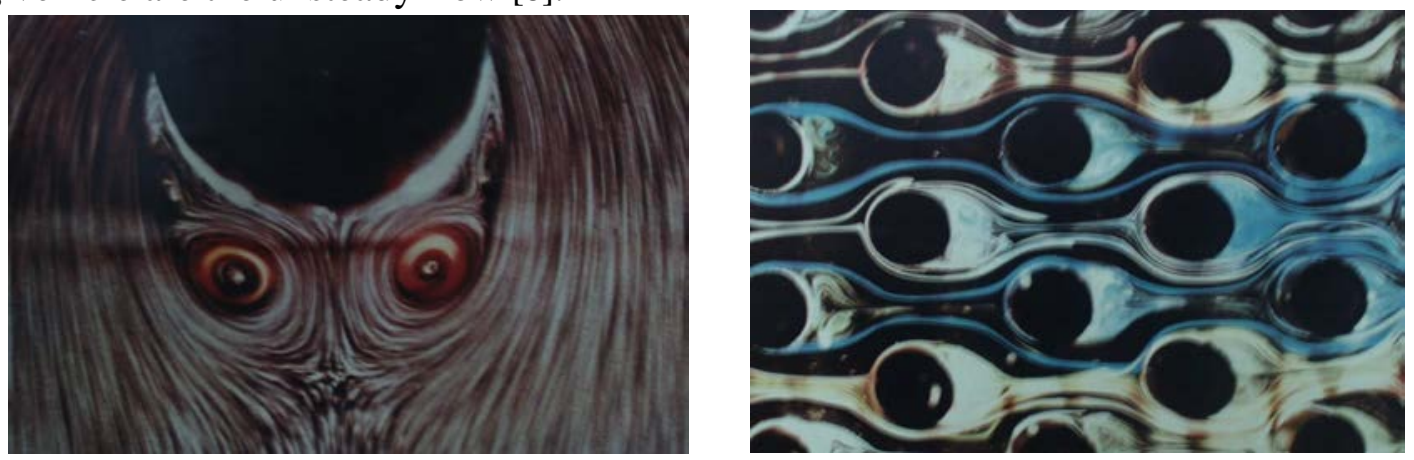

Fig.2 Steady Flow and Unsteady Flow

The free stream flow is the air in a region where pressure, temperature and relative velocity are unaffected by the passage of the aircraft through it (see Fig 3). It is also sometimes called relative airflow (RAF). Streamline is the term given to the path traced by a particle in a steady fluid flow [9].

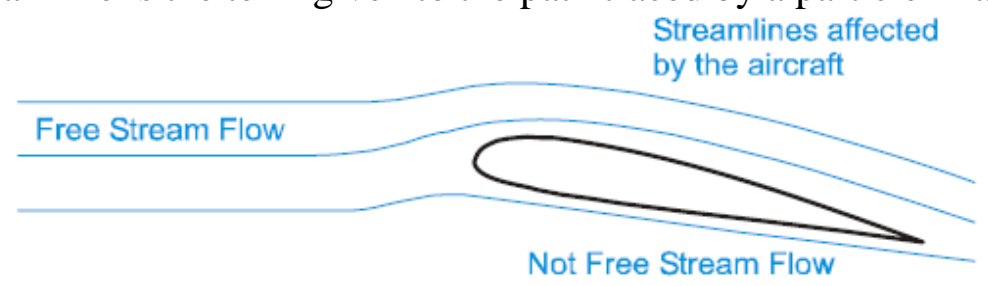

Fig.3 Free Stream Flow and Streamline

Assuming that the flow is constant, the two dimensional incompressible N-S equations are written as:

$$
\begin{aligned}
& u \frac{\partial u}{\partial x}+v \frac{\partial u}{\partial y}=-\frac{1}{\rho} \frac{\partial p}{\partial x}+\frac{\mu}{\rho}\left(\frac{\partial^{2} u}{\partial x^{2}}+\frac{\partial^{2} v}{\partial y^{2}}\right) \\
& u \frac{\partial v}{\partial x}+v \frac{\partial v}{\partial y}=-\frac{1}{\rho} \frac{\partial p}{\partial y}+\frac{\mu}{\rho}\left(\frac{\partial^{2} v}{\partial x^{2}}+\frac{\partial^{2} v}{\partial y^{2}}\right)
\end{aligned}
$$

\section{Laminar Boundary Layer and Turbulent Boundary Layer}

In fact, the study also found that the same is in the boundary layer, the flow is not exactly the same, 
there are laminar boundary layer and turbulent boundary layer. According to its flow features, in the laminar boundary layer, the air flows in laminas, without confusion of all the laminas or strong up and down turbulence of the air fluid particles; in the turbulent boundary layer, there is obvious up and down turbulence of the air fluid particles and strong mixture of all the laminas, with small local vortex and pulse of velocity, which is a turbulent flow.

The thicknesses of laminar boundary layer and turbulent boundary layer are different and the distribution of their velocities is also different [10]. In the turbulent boundary layer, there is strong up and down turbulence of the air fluid particles and the exchange of momentum between all the layers is increased, so the thickness of the turbulent boundary layer is big; the flow rate difference between the adjacent laminas will be reduced in the layer, so the velocity gradient along the normal direction is decreased [11]. However, the up and down turbulence of the air fluid particles is restricted on the part of the turbulent boundary layer close to the object surface, so the flow is still the laminar flow, called the laminar bottom layer. The flow rate difference at the laminar bottom layer is bigger than that of the laminar boundary layer, so the velocity gradient of the turbulent boundary layer on the object surface is bigger than that of the laminar one, that is, $(\mathrm{d} V / \mathrm{d} y)_{h=0 \text { turbulent }}>(\mathrm{d} V / \mathrm{d} y)_{h=0 \text { laminar }}$.

\section{Separation of the Boundary Layer}

Air flow through the wing surface, the upper surface of the pipe is the first contraction, and then to the smallest area and then slow expansion, the lower surface of the flow pipe cross-sectional area is not much change. The contraction section of the pipe cross-sectional area gradually reduced, according to the boundary continuous equation and the Bernoulli equation concluded that: contraction section of the pipe cross-sectional area is gradually reduced, speed will gradually increasing, and pressure will gradually decrease. In this section to take a control body to analyze, and to enlarge the control body, from the left to the right side, along the flow direction static pressure in the reduction. That is to say the left side pressure small, this will produce a pressure difference, the pressure difference is in the direction of pointing right here, which is along the direction of flow, it plays a role in the acceleration. So, we call this section along the pressure gradient section. The pressure is gradually reduced, that is, the pressure gradient is less than zero.

This is in the contraction section, the same, in the expansion section: the flow tube cross-sectional area increases, the flow rate will slow down, the static pressure rise. Also take a control body and enlarge. Increases from left to right and the static pressure along the flow direction, that is to say the right small hydrostatic pressure, this will produce a pressure difference, but the difference of pressure and flow in the opposite direction, is against the flow, to reduce, so it is called the inverse pressure gradient. At this time the pressure gradually increased, the pressure gradient is greater than zero. The flow tube is the smallest, the flow rate is the fastest, the pressure is the minimum, in the middle of this place is called the minimum pressure point. Pressure gradient is zero.

In this way, on the face of the upper wing, the upper wing of the main flow tube, the minimum pressure point as the boundary, is divided into the pressure gradient section and the reverse pressure gradient section. The boundary layer is also the same, the pressure of each point along the normal direction is equal, and is equal to the main pressure. Therefore, the normal of the minimum pressure point, also can be divided into the boundary layer of the pressure gradient section and the reverse pressure gradient section. Numerical simulation results of surface pressure distribution in the boundary layer are shown in Figure 4. 


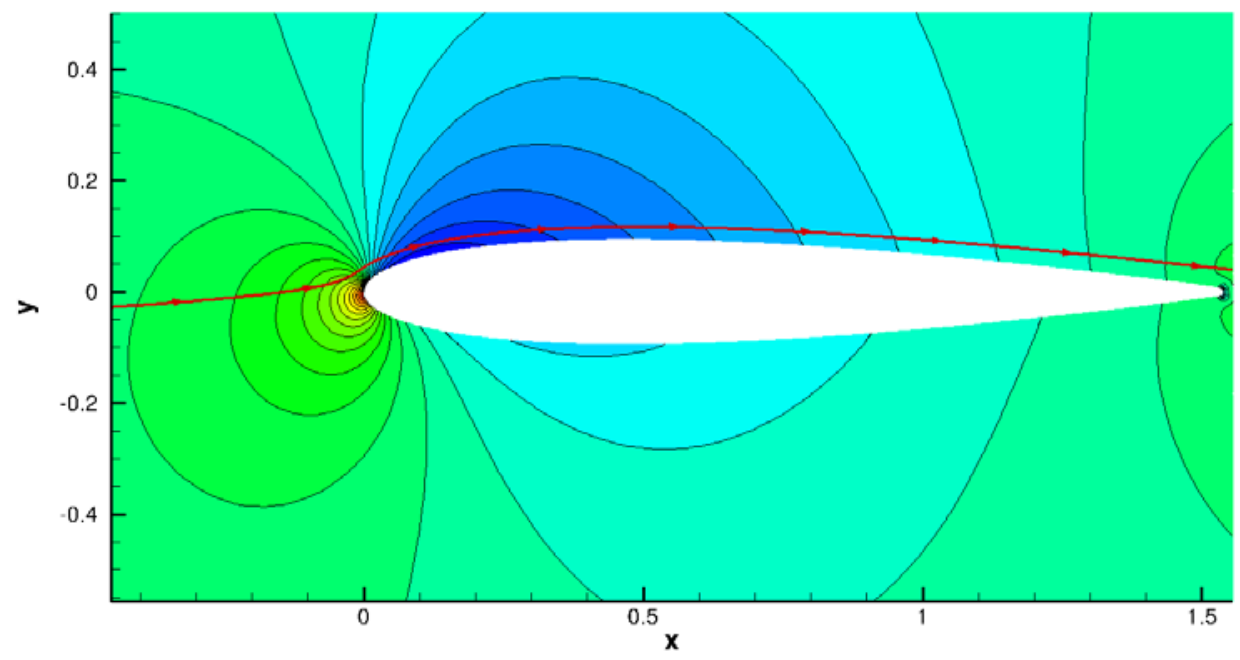

Fig.4 Numerical simulation results of surface pressure distribution in the boundary layer

On the left side of the pressure gradient section, along with the pressure to accelerate the role, despite the friction, the pressure difference will continue to push the bottom of the air micro clusters along the main flow. At the lowest point of the pressure is still a certain speed, rely on inertia and then continue to move forward. After a minimum pressure point, the boundary layer flow by the dual role of inverse pressure and friction, two are resistance, no power, so that the underlying flow boundary layer is quickly slow down, even in the stop point $\mathrm{S}$ on a certain position, speed decreases to zero. Local flow from high pressure areas to low pressure is speed up. So the air in the inverse pressure state start back, so, at after the two streams meet, more and more air accumulation, the two streams will arch from the surface of the wing. And the mainstream of the air flow interactions, the formation of a large number of whirlpool. That is to say that the point of separation of the turbulent boundary layer lies behind more than that of the laminar one.

\section{Conclusion}

The boundary layer is a thin layer of air viscosity, which is different from the ideal fluid. The boundary layer is divided into the laminar boundary layer and the turbulent boundary layer according to the Reynolds number. According to the boundary layer numerical simulation results show that the boundary layer is in the presence of the pressure gradient section and the reverse pressure gradient section. The reverse pressure gradient and air viscosity are the fundamental reasons for the separation of boundary layer flow.

\section{References}

[1] YuXin Zhao, ShiHe Yi, LiFeng Tian, Lin He, ZhongYu Cheng. Multiresolution analysis of density fluctuation in supersonic mixing layer $[\mathrm{J}]$. Science China Technological Sciences . 2010

[2] LiFeng Tian, ShiHe Yi, YuXin Zhao, Lin He, ZhongYu Cheng. Study of density field measurement based on NPLS technique in supersonic flow [J]. Science in China Series G: Physics, Mechanics and Astronomy . 2009

[3] M. W. Smith, A. J. Smits. Visualization of the structure of supersonic turbulent boundary layers [J]. Experiments in Fluids . 1995 (4)

[4] Anh-Tuan Le. A numerical study of three-dimensional turbulent boundary layers [D]. University of California, Los Angeles, 1999.

[5]Stephen Guarini. Direct numerical simulation of supersonic turbulent boundary layers [D]. Stanford University, 1998. 
[6] SHTESSEL Y B,BUFFINGTON J M,BANDA S. S. Multiple time scale flight control using reconfigurable sliding modes. Journal of Guidance Control and Dynamics. 1999

[7] MACRO D B,HEALEY A J. Command control and experiment results with the NPS ARIES AUVs. IEEE Journal of Oceanic Engineering . 2001

[8] WANG Bing, ZHANG Huiqiang, CAO Hongjun. Flow Dynamics of a Spiral-groove Dry-gas Seal [J]. Chinese Journal of Mechanical Engineering. 2013(01)

[9] Bretherton, Christopher S, Park, Sungsu. A New Moist Turbulence Parameterization in the Community Atmosphere Model [J]. Journal of Climate . 2009 (12)

[10] Hu, Xiao-Ming, Nielsen-Gammon, John W, Zhang, Fuqing. Evaluation of Three Planetary Boundary Layer Schemes in the WRF Model [J]. Journal of Applied Meteorology and Climatology . 2010 (9)

[11] HUANG Zhangfeng1, ZHOU Heng1, 2 \& LUO Jisheng1,2 1. Department of Mechanics, Tianjin University, Tianjin 300072, China; 2. Liu-Hui Center of Applied Mathematics, Nankai University and Tianjin University, Tianjin 300072, China. Direct numerical simulation of a supersonic turbulent boundary layer on a flat plate and its analysis [J]. Science in China(Series G:Physics,Mechanics \& Astronomy). 2005(05) 